\title{
RIG-I like receptors
}

Natalie A. Borg 1*

\begin{abstract}
RIG-I (retinoic-acid inducible gene I, also known as DDX 58 ) is the best characterized receptor within the RIG-I like receptor (RLR) family. Together with MDA 5 (melanoma differentiation-associated 5) and LGP2 (laboratory of genetics and physiology 2), this family of cytoplasmic pattern recognition receptors (PRRs) are sentinels for intracellular viral RNA that is a product of viral infection. The RLR receptors provide frontline defence against viral infections in most tissues.
\end{abstract}

\section{RLR ligands}

The RIG-I receptor prefers to bind short ( $<2000 \mathrm{bp}$ ) single- or double-stranded RNA carrying an uncapped $5^{\prime}$ triphosphate and additional motifs such as poly-uridine rich RNA motifs. ${ }^{[1][2]}$ RIG-I triggers an immune response to RNA viruses from various families including the paramyxoviruses (e.g. measles), rhabdoviruses (e.g. vesicular stomatitis virus) and orthomyxoviruses (e.g. influenza A). ${ }^{[3][4][5][6][7]}$ MDA5 ligands are poorly characterized, but the preference is for long double-stranded RNA (>2000 bp), such as the replicative form of picornavirus RNA that is found in picornavirus-infected cells. ${ }^{[8][9]}$ LGP2 binds to blunt-ended double-stranded RNA of variable length, ${ }^{[10][11]}$ and also to RNA-bound MDA5 to regulate filament formation. ${ }^{[12]}$ The latter is linked to LGP2's recognition of picornaviruses (e.g. encephalomyocarditis virus), as per MDA5. ${ }^{[13]}$

\section{Structural features of the RLR receptors}

The RLR receptors are members of the DEAD-box helicase family (despite containing a DExD/H motif, rather than the DEAD motif characteristic of the family) and share a common domain architecture. All contain a catalytic helicase core made up of two RecA-like domains (Figure 1A). The catalytic helicase core contains at least

${ }^{1}$ Infection \& Immunity Program, Monash Biomedicine Discovery Institute and Department of Biochemistry and Molecular Biology, Monash University, Clayton, Victoria, Australia.

*Author correspondence: natalie.borg@monash.edu

ORCID: 0000-0002-8677-9056

Licensed under: CC-BY

Received 15-09-2018; accepted 11-03-2019
9 highly conserved sequence motifs that coordinate ATP and RNA binding and the hydrolysis of ATP to unwind RNA. A C-terminal domain (CTD) follows the helicase core and this domain also binds viral RNA (Figure $1 A, 1 B)$. Distinct RNA-binding loops within the CTD of the three RLRs dictate the type of RNA that they can bind. ${ }^{[14]}$ In addition to the helicase core and CTD, RIG-I and MDA5 have two $\mathrm{N}$-terminal CARD (caspase active recruitment domains) that are essential to the initiation of downstream signaling (Figure 1A). LGP2 is dissimilar to both RIG-I and MDA5 as it lacks the CARD signaling domains and instead is implicated as a positive and negative regulator of RIG-I and MDA5. [15][16][17][18][19][20][21]
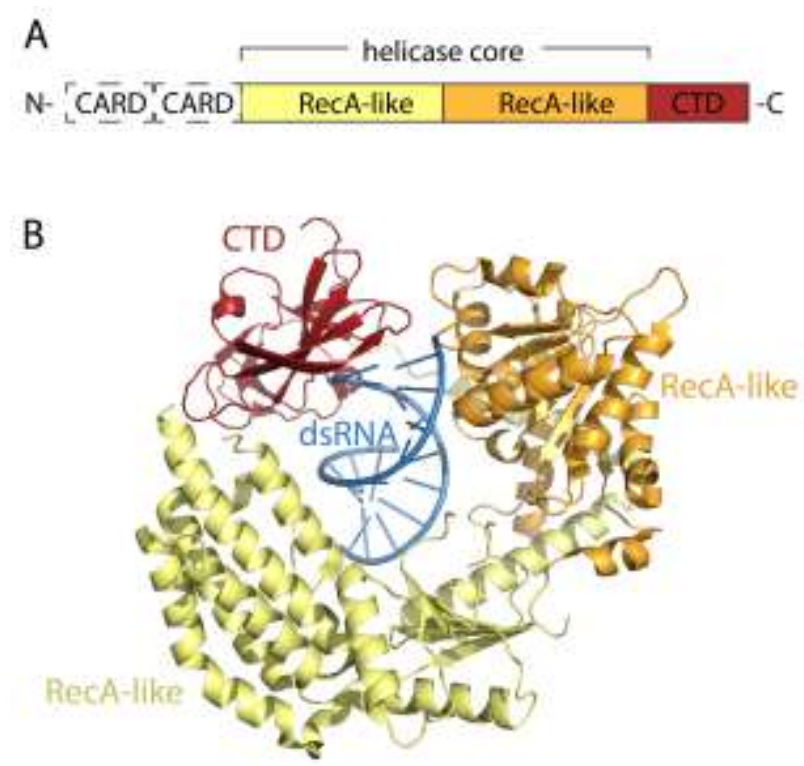

Figure 1 | RIG-I domain architecture. (A) Schematic representation of full-length RIG-I. (B) X-ray crystal structure of RNAbound RIG-I (PDB: 2YKG), excluding the CARD domains. 


\section{Activation of RLR signaling}

In uninfected cells that are absent of viral RNA RIG-I exists in an inactive conformation in which the CARD domains are masked due to their interaction with the CTD. ${ }^{[22]}$ Upon binding RNA, RIG-I changes into a conformation in which the CARD domains are exposed and 'available' for signaling. Conversely, the MDA5 CARDs are unhindered in the absence of viral RNA. ${ }^{\left[{ }^{23]}\right.}$ As a safeguard for RLR activation, the exposed RIG-I and MDA5 CARDs can undergo post-translational modifications (e.g. ubiquitination, phosphorylation) that either positively or negatively regulate downstream signaling.

\section{RIG-I antiviral signaling}

In the activated state the exposed RIG-I CARD domains interact with the CARD domains of MAVS (mitochondrial antiviral signaling protein, also known as IPS-1, VISA or (ardif) which sits on the outer surface of the mitochondria (Figure 2). This binding event is essential to signaling as it causes MAVS to form large functional aggregates in which TRAF3 (TNF receptor-associated factor 3) and subsequently the IKKe/TBK1 (I-kappa-B kinase-epsilon/TANK-binding kinase 1 ) complex are recruited. The IKK $\varepsilon / T B K 1$ complex leads to the activation of the transcription factors interferon regulatory factor

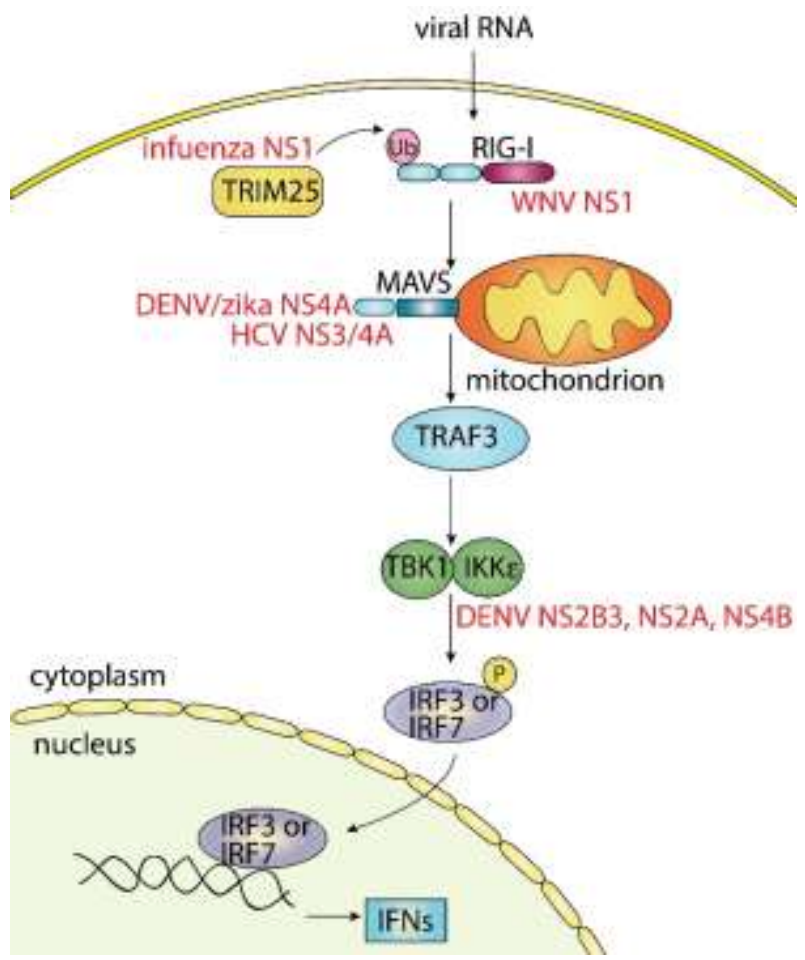

Figure 2 | Schematic of RLR signaling. Ub denotes ubiquitination, $\mathrm{P}$ denotes phosphorylation.
3 (IRF3) and IRF7 which induce type I (including IFN $\alpha$ and IFN $\beta$ ) and type III interferons (IFN) (Figure 2). The type I IFNs bind type I IFN receptors on the surface of the cell that produced them, and also other cell types that express the receptor, to activate JAK-STAT (Janus kinase/signal transducers and activators of transcription) signaling. This leads to the induction of hundreds of interferon stimulated genes (ISGs) that amplify the IFN response. Overall this causes the death of infected cells, the protection of surrounding cells and the activation of the antigen-specific antiviral immune response. Collectively this coordinated antiviral immune response controls the viral infection.

\section{Regulation of RLR signalling}

As prolonged IFN production is linked to human disease RLR signaling must be tightly regulated. One of various ways that this is achieved is by post-translationally modifying, or tagging, host RLR signaling proteins with phosphate (known as phosphorylation) or ubiquitin (known as ubiquitination). These tags can also be removed, which adds an additional regulatory layer to RLR signaling. These post-translational modifications, and their removal, are prevalent in RLR signaling and even regulate the RIG-I receptor itself. Most famously the RIG-I CARD domain is phosphorylated by protein kinase $C-\alpha(P K C-\alpha)$ and PKC- $\beta$ in the resting state to negatively regulate signaling. ${ }^{[24][25][26]}$ Upon viral infection RIG-I is dephosphorylated by PP $1 \alpha$ and $P P 1 \gamma_{1}{ }^{[27]}$ permitting the ubiquitination of the RIG-ICARD domain by the E3 ligase TRIM25 to activate the RLR-mediated antiviral immune response. ${ }^{[28]}$ Given post-translational modifications are so pertinent to the activation of RLR signaling, it is not surprising that they are directly, or indirectly, targeted by viruses such as influenza $A^{[29]}$ and measles, ${ }^{[30]}$ respectively, to suppress signaling (Figure 2).

\section{Viral hijacking of RLR signaling}

Viruses have evolved ways to subvert RLR signaling to enhance their survival. For example, influenza $A$ virus and West Nile virus (WNV) use their NS1 (nonstructural protein 1) proteins to block RIG-I ubiquitination by TRIM25, or cause RIG-I degradation, respectively, which in turn inhibits IFN production (Figure 2). ${ }^{\text {29][31] }}$ This outcome is also achieved by the hepatitis $C(\mathrm{HCV})$ NS3/4A protein by cleaving a part of MAVS (Figure 2), ${ }^{[32]}$ and the foot-and-mouth disease virus (FMDV) leader protease (Lpro) which cleaves LGP2. ${ }^{[33]}$ Likewise, dengue virus (DENV) uses its NS2B3, NS2A and NS4B pro- 
teins to bind IKKE and prevent IRF3 phosphorylation $^{[34][35]}$ and its NS4A protein, as per the zika virus, to bind MAVS to block RLR receptor binding. ${ }^{[36][37]}$ Another prominent example is that of the paramyxovirus $\checkmark$ proteins, which directly bind various RLR or downstream signaling proteins including MDA5, LGP2, and STAT, ${ }^{[38][39][40]}$ or proteins such as PP1 $\alpha$ and PP1 $\gamma^{[30]}$ that negatively regulate RLR signaling (Figure 2 ).

\section{Summary}

The RLR receptors are cytoplasmic PRRs that provide frontline defence against prominent viral infections such as influenza $A$ and measles. RLR signaling produces proinflammatory cytokines and type I and type III interferons (IFN) and orchestrates the induction of ISGs via the JAK-STAT signalling pathway to establish an antiviral state that controls infection. The RLR signaling cascade is heavily regulated by post-translational modifications. Viral proteins also target proteins within the cascade to enhance virus survival.

\section{Additional information}

\section{Competing interests}

The author has no competing interests

\section{References}

1. Kato, Hiroki; Takeuchi, Osamu; Mikamo-Satoh, Eriko; Hirai, Reiko; Kawai, Tomoji; Matsushita, Kazufumi; Hiiragi, Akane; Dermody, Terence S. et al. (2008-06-30). "Length-dependent recognition of double-stranded ribonucleic acids by retinoic acid-inducible gene-I and melanoma differentiation-associated gene 5". The Journal of Experimental Medicine205 (7): 1601-1610. doi:10.1084/jem.20080091. ISSN 0022-1007.

2. Saito, Takeshi; Owen, David M.; Jiang, Fuguo; Marcotrigiano, Joseph; Gale, Michael (2008-06-11). "Innate immunity induced by compositiondependent RIG-I recognition of hepatitis C virus RNA". Nature 454 (7203): 523-527. doi:10.1038/nature07106. ISSN 0028-0836.

3. Baum, A.; Sachidanandam, R.; Garcia-Sastre, A. (2010-08-30). "Preference of RIG-I for short viral RNA molecules in infected cells revealed by nextgeneration sequencing". Proceedings of the National Academy of Sciences 107 (37): 16303-16308. doi:10.1073/pnas.1005077107. ISSN 0027-8424.

4. Gitlin, L.; Barchet, W.; Gilfillan, S.; Cella, M.; Beutler, B.; Flavell, R. A.; Diamond, M. S.; Colonna, M. (2006-05-19). "Essential role of mda-5 in type I IFN responses to polyriboinosinic:polyribocytidylic acid and encephalomyocarditis picornavirus". Proceedings of the National Academy of Sciences 103 (22): 8459-8464. doi:10.1073/pnas.0603082103. ISSN 0027-8424.

5. Hornung, V.; Ellegast, J.; Kim, S.; Brzozka, K.; Jung, A.; Kato, H.; Poeck, H.; Akira, S. et al. (2006-11-10). "5'-Triphosphate RNA Is the Ligand for RIG-I". Science 314 (5801): 994-997. doi:10.1126/science.1132505. ISSN 00368075.

6. Wang, Haifeng; Ryu, Wang-Shick (2010-07-15). "Hepatitis B Virus Polymerase Blocks Pattern Recognition Receptor Signaling via Interaction with DDX3: Implications for Immune Evasion". PLoS Pathogens 6 (7): e1000986. doi:10.1371/journal.ppat.1000986. ISSN 1553-7374.

7. Schlee, Martin; Roth, Andreas; Hornung, Veit; Hagmann, Cristina Amparo; Wimmenauer, Vera; Barchet, Winfried; Coch, Christoph; Janke, Markus et al. (2009-07). "Recognition of 5' Triphosphate by RIG-I Helicase Requires Short Blunt Double-Stranded RNA as Contained in Panhandle of Negative-
Strand Virus". Immunity 31 (1): 25-34. doi:10.1016/j.immuni.2009.05.008. ISSN 1074-7613.

8. Feng, Qian; Hato, Stanleyson V.; Langereis, Martijn A.; Zoll, Jan; VirgenSlane, Richard; Peisley, Alys; Hur, Sun; Semler, Bert L. et al. (2012-11). "MDA5 Detects the Double-Stranded RNA Replicative Form in Picornavirus-Infected Cells". Cell Reports 2 (5): 1187-1196. doi:10.1016/j.celrep.2012.10.005. ISSN 2211-1247.

9. Kato, Hiroki; Takeuchi, Osamu; Sato, Shintaro; Yoneyama, Mitsutoshi; Yamamoto, Masahiro; Matsui, Kosuke; Uematsu, Satoshi; Jung, Andreas et al. (2006-04-09). "Differential roles of MDA5 and RIG-I helicases in the recognition of RNA viruses". Nature 441 (7089): 101-105. doi:10.1038/nature04734. ISSN 0028-0836.

10. Li, Xiaojun; Ranjith-Kumar, C. T.; Brooks, Monica T.; Dharmaiah, S.; Herr, Andrew B.; Kao, Cheng; Li, Pingwei (2009-03-11). "The RIG-I-like Receptor LGP2 Recognizes the Termini of Double-stranded RNA". Journal of Biological Chemistry 284 (20): 13881-13891. doi:10.1074/jbc.m900818200. ISSN 0021-9258.

11. Murali, Ayaluru; Li, Xiaojun; Ranjith-Kumar, C. T.; Bhardwaj, Kanchan; Holzenburg, Andreas; Li, Pingwei; Kao, C. Cheng (2008-04-14). "Structure and Function of LGP2, a DEX(D/H) Helicase That Regulates the Innate Immunity Response". Journal of Biological Chemistry 283 (23): 1582515833. doi:10.1074/jbc.m800542200. ISSN 0021-9258.

12. Bruns, Annie M.; Leser, George P.; Lamb, Robert A.; Horvath, Curt M. (2014-09). "The Innate Immune Sensor LGP2 Activates Antiviral Signaling by Regulating MDA5-RNA Interaction and Filament Assembly". Molecular Cell 55 (5): 771-781. doi:10.1016/j.molcel.2014.07.003. ISSN 1097-2765.

13. Satoh, T.; Kato, H.; Kumagai, Y.; Yoneyama, M.; Sato, S.; Matsushita, K.; Tsujimura, T.; Fujita, T. et al. (2010-01-08). "LGP2 is a positive regulator of RIG-I- and MDA5-mediated antiviral responses". Proceedings of the National Academy of Sciences 107 (4): 1512-1517. doi:10.1073/pnas.0912986107. ISSN 0027-8424.

14. Takahasi, Kiyohiro; Kumeta, Hiroyuki; Tsuduki, Natsuko; Narita, Ryo; Shigemoto, Taeko; Hirai, Reiko; Yoneyama, Mitsutoshi; Horiuchi, Masataka et al. (2009-04-20). "Solution Structures of Cytosolic RNA Sensor MDA5 and LGP2 C-terminal Domains". Journal of Biological Chemistry 284 (26): 17465-17474. doi:10.1074/jbc.m109.007179. ISSN 0021-9258.

15. Bruns, Annie M.; Leser, George P.; Lamb, Robert A.; Horvath, Curt M. (2014-09). "The Innate Immune Sensor LGP2 Activates Antiviral Signaling by Regulating MDA5-RNA Interaction and Filament Assembly". Molecular Cell 55 (5): 771-781. doi:10.1016/j.molcel.2014.07.003. ISSN 1097-2765.

16. Childs, Kay S.; Randall, Richard E.; Goodbourn, Stephen (2013-05-09). "LGP2 Plays a Critical Role in Sensitizing mda-5 to Activation by DoubleStranded RNA". PLOS ONE 8(5): e64202. doi:10.1371/journal.pone.0064202. ISSN 1932-6203.

17. Komuro, A.; Horvath, C. M. (2006-10-04). "RNA- and Virus-Independent Inhibition of Antiviral Signaling by RNA Helicase LGP2". Journal of Virology 80 (24): 12332-12342. doi:10.1128/jvi.01325-06. ISSN 0022-538X.

18. Parisien, Jean-Patrick; Lenoir, Jessica J; Mandhana, Roli; Rodriguez, Kenny R; Qian, Kenin; Bruns, Annie M; Horvath, Curt M (2018-04-16). "RNA sensor LGP2 inhibits TRAF ubiquitin ligase to negatively regulate innate immune signaling". EMBO reports 19 (6): e45176. doi:10.15252/embr.201745176. ISSN 1469-221X.

19. Saito, T.; Hirai, R.; Loo, Y.-M.; Owen, D.; Johnson, C. L.; Sinha, S. C.; Akira, S.; Fujita, T. et al. (2006-12-26). "Regulation of innate antiviral defenses through a shared repressor domain in RIG-I and LGP2". Proceedings of the National Academy of Sciences 104 (2): 582-587. doi:10.1073/pnas.0606699104. ISSN 0027-8424.

20. Satoh, T.; Kato, H.; Kumagai, Y.; Yoneyama, M.; Sato, S.; Matsushita, K.; Tsujimura, T.; Fujita, T. et al. (2010-01-08). "LGP2 is a positive regulator of RIG-I- and MDA5-mediated antiviral responses". Proceedings of the National Academy of Sciences 107 (4): 1512-1517. doi:10.1073/pnas.0912986107. ISSN 0027-8424.

21. Uchikawa, Emiko; Lethier, Mathilde; Malet, Hélène; Brunel, Joanna; Gerlier, Denis; Cusack, Stephen (2016-05). "Structural Analysis of dsRNA Binding to Anti-viral Pattern Recognition Receptors LGP2 and MDA5". Molecular Cell 62 (4): 586-602. doi:10.1016/j.molcel.2016.04.021. ISSN 1097-2765.

22. Luo, Dahai; Ding, Steve C.; Vela, Adriana; Kohlway, Andrew; Lindenbach, Brett D.; Pyle, Anna Marie (2011-10). "Structural Insights into RNA Recognition by RIG-I". Cell 147 (2): 409-422. doi:10.1016/j.cell.2011.09.023. ISSN 0092-8674.

23. Berke, Ian $C_{i}$ Modis, Yorgo (2012-02-07). "MDA5 cooperatively forms dimers and ATP-sensitive filaments upon binding double-stranded RNA". The EMBO Journal 31 (7): 1714-1726. doi:10.1038/emboj.2012.19. ISSN 0261-4189. 
24. Gack, M. U.; Nistal-Villan, E.; Inn, K.-S.; Garcia-Sastre, A.; Jung, J. U. (201001-13). "Phosphorylation-Mediated Negative Regulation of RIG-I Antiviral Activity". Journal of Virology 84 (7): 3220-3229. doi:10.1128/jvi.02241-09. ISSN 0022-538X

25. Maharaj, N. P.; Wies, E.; Stoll, A.; Gack, M. U. (2011-11-23). "Conventional Protein Kinase C- (PKC-) and PKC- Negatively Regulate RIG-I Antiviral Signal Transduction". Journal of Virology 86 (3): 1358-1371. doi:10.1128/jvi.06543-11. ISSN 0022-538X

26. Nistal-Villán, Estanislao; Gack, Michaela U.; Martínez-Delgado, Gustavo; Maharaj, Natalya P.; Inn, Kyung-Soo; Yang, Heyi; Wang, Rong; Aggarwal, Aneel K. et al. (2010-04-20). "Negative Role of RIG-I Serine 8 Phosphorylation in the Regulation of Interferon- $\beta$ Production". Journal of Biological Chemistry $285 \quad$ (26): 20252-20261. doi:10.1074/jbc.m109.089912. ISSN 0021-9258.

27. Wies, Effi; Wang, May K.; Maharaj, Natalya P.; Chen, Kan; Zhou, Shenghua; Finberg, Robert W.; Gack, Michaela U. (2013-03). "Dephosphorylation of the RNA Sensors RIG-I and MDA5 by the Phosphatase PP1 Is Essential for Innate Immune Signaling". Immunity 38(3): 437-449. doi:10.1016/j.immuni.2012.11.018. ISSN 1074-7613.

28. Gack, Michaela U.; Shin, Young C.; Joo, Chul-Hyun; Urano, Tomohiko; Liang, Chengyu; Sun, Lijun; Takeuchi, Osamu; Akira, Shizuo et al. (200703-28). "TRIM25 RING-finger E3 ubiquitin ligase is essential for RIG-Imediated antiviral activity". Nature 446 (7138): 916-920. doi:10.1038/nature05732. ISSN 0028-0836.

29. Gack, Michaela Ulrike; Albrecht, Randy Allen; Urano, Tomohiko; Inn, Kyung-Soo; Huang, I-Chueh; Carnero, Elena; Farzan, Michael; Inove, Satoshi et al. (2009-05). "Influenza A Virus NS1 Targets the Ubiquitin Ligase TRIM25 to Evade Recognition by the Host Viral RNA Sensor RIG-I". Cell Host \& Microbe 5 (5): 439-449. doi:10.1016/j.chom.2009.04.006. ISSN 1931-3128.

30. Davis, Meredith E.; Wang, May K.; Rennick, Linda J.; Full, Florian; Gableske, Sebastian; Mesman, Annelies W.; Gringhuis, Sonja I.; Geijtenbeek, Teunis B.H. et al. (2014-07). "Antagonism of the Phosphatase PP1 by the Measles Virus V Protein Is Required for Innate Immune Escape of MDA5". Cell Host \& Microbe 16 (1): 19-30. doi:10.1016/j.chom.2014.06.007. ISSN 1931-3128.

31. Zhang, Hong-Lei; Ye, Han-Qing; Liu, Si-Qing; Deng, Cheng-Lin; Li, XiaoDan; Shi, Pei-Yong; Zhang, Bo (2017-06-28). "West Nile Virus NS1 Antagonizes Interferon Beta Production by Targeting RIG-I and MDA5". Journal of Virology 91 (18). doi:10.1128/jvi.02396-16. ISSN 0022-538X.

32. Li, X.-D.; Sun, L.; Seth, R. B.; Pineda, G.; Chen, Z. J. (2005-11-21). "Hepatitis $\mathrm{C}$ virus protease NS3/4A cleaves mitochondrial antiviral signaling protein off the mitochondria to evade innate immunity". Proceedings of the National Academy of Sciences 102 (49): 17717-17722. doi:10.1073/pnas.0508531102. ISSN 0027-8424.

33. Rodríguez Pulido, Miguel; Sánchez-Aparicio, María Teresa; MartínezSalas, Encarnación; García-Sastre, Adolfo; Sobrino, Francisco; Sáiz, Margarita (2018-06-29). "Innate immune sensor LGP2 is cleaved by the Leader protease of foot-and-mouth disease virus". PLOS Pathogens 14 (6): e1007135. doi:10.1371/journal.ppat.1007135. ISSN 1553-7374.

34. Angleró-Rodríguez, Yesseinia I.; Pantoja, Petraleigh; Sariol, Carlos A. (2013-10-30). "Dengue Virus Subverts the Interferon Induction Pathway via NS2B/3 Protease-IKB Kinase $\varepsilon$ Interaction". Clinical and Vaccine Immunology 21 (1): 29-38. doi:10.1128/cvi.00500-13. ISSN 1556-6811.

35. Dalrymple, Nadine A.; Cimica, Velasco; Mackow, Erich R. (2015-05-12). "Dengue Virus NS Proteins Inhibit RIG-I/MAVS Signaling by Blocking TBK1/IRF3 Phosphorylation: Dengue Virus Serotype 1 NS4A Is a Unique Interferon-Regulating Virulence Determinant". mBio $6 \quad$ (3). doi:10.1128/mbio.00553-15. ISSN 2150-7511.

36. He, Zhenjian; Zhu, Xun; Wen, Weitao; Yuan, Jie; Hu, Yiwen; Chen, Jiahui; An, Shu; Dong, Xinhuai et al. (2016-06-01). "Dengue Virus Subverts Host Innate Immunity by Targeting Adaptor Protein MAVS". Journal of Virology 90 (16): 7219-7230. doi:10.1128/jvi.00221-16. ISSN 0022-538X.

37. Ma, Jinzhu; Ketkar, Harshada; Geng, Tingting; Lo, Emily; Wang, Leilei; Xi, Juemin; Sun, Qiangming; Zhu, Zhanbo et al. (2018-06-25). "Zika Virus Non-structural Protein 4A Blocks the RLR-MAVS Signaling". Frontiers in Microbiology 9. doi:10.3389/fmicb.2018.01350. ISSN 1664-302X.

38. Andrejeva, J.; Childs, K. S.; Young, D. F.; Carlos, T. S.; Stock, N.; Goodbourn, S.; Randall, R. E. (2004-11-24). "The V proteins of paramyxoviruses bind the IFN-inducible RNA helicase, mda-5, and inhibit its activation of the IFN-promoter". Proceedings of the National Academy of Sciences 101 (49): 17264-17269. doi:10.1073/pnas.0407639101. ISSN 0027-8424.

39. Childs, Kay; Stock, Nicola; Ross, Craig; Andrejeva, Jelena; Hilton, Louise; Skinner, Michael; Randall, Richard; Goodbourn, Stephen (2007-03). "mda5 , but not RIG-I, is a common target for paramyxovirus V proteins". Virology 359 (1): 190-200. doi:10.1016/j.virol.2006.09.023. ISSN 0042-6822.

40. Rodriguez, Kenny R.; Horvath, Curt M. (2014-05-14). "Paramyxovirus V Protein Interaction with the Antiviral Sensor LGP2 Disrupts MDA5 Signaling Enhancement but Is Not Relevant to LGP2-Mediated RLR Signaling Inhibition". Journal of Virology 88 (14): 8180-8188. doi:10.1128/jvi.00737-14. ISSN 0022-538X. 